\title{
Multiprocessor Energy-Efficient Scheduling for Real-Time Tasks with Different Power Characteristics*
}

\author{
Jian-Jia Chen and Tei-Wei Kuo \\ Department of Computer Science and Information Engineering \\ Graduate Institute of Networking and Multimedia \\ National Taiwan University, Taipei, Taiwan, ROC \\ Email: $\{\mathrm{r} 90079, \mathrm{ktw}\} @$ csie.ntu.edu.tw
}

\begin{abstract}
In the past decades, a number of research results have been reported for energy-efficient scheduling over uniprocessor and multiprocessor environments. Different from many of the past results on the assumption for task power characteristics, we consider real-time scheduling of tasks with different power characteristics. The objective is to minimize the energy consumption of task executions under the given deadline constraint. When tasks have a common deadline and are ready at time 0 , we propose an optimal real-time task scheduling algorithm for multiprocessor environments with the allowance of task migration. When no task migration is allowed, a 1.412approximation algorithm for task scheduling is proposed for different settings of power characteristics. The performance of the approximation algorithm was evaluated by an extensive set of experiments, where excellent results were reported.
\end{abstract}

\section{Introduction}

With the advanced technology in VLSI circuit designs, many modern processors could now operate at various supply voltages, where different supply voltages lead to different processing speeds. Many computer systems, especially embedded systems, adopt not only voltage-scaling processors but also various energy-efficient strategies in managing their subsystems intelligently. Beside the energy-efficiency designs for battery-powered systems, how to reduce energy consumption for multi-processor systems, such as server farms, also receives a lot of attention in the past decade. As pointed out in [1], multiprocessor implementations of real-time systems could be more energy-efficient than uniprocessor implementations, due to the convex power consumption functions.

Energy-efficient scheduling is to derive a schedule for realtime tasks with minimization on the energy consumption such

* Support in parts by research grants from ROC National Science Council NSC-93-2752-E-002-008-PAE and NSC-93-2218-E-002-140. that the timing constraints can be met. The considerations of timing constraints in task scheduling significantly complicate the problems in energy-efficient scheduling. Uniprocessor energy-efficient scheduling problems have been widely explored, e.g., $[2,3,10-12,19]$. The energy-efficient realtime task scheduling problems over multiprocessors are often $\mathcal{N} \mathcal{P}$-hard. When all of the power consumption functions of tasks are the same, Chen, et al. [5,18] proposed approximation algorithms to schedule frame-based tasks over multiprocessors with and without independent voltage scalings, where all the tasks share a common deadline and arrive at the same time. In $[7,8,20]$, energy-efficient scheduling algorithms based on list heuristics were proposed to schedule real-time tasks with precedence constraints. Mishra, et al. [13] explored energy-efficient scheduling issues with the considerations of task communication delay.

This work is motivated by the energy-efficient scheduling of tasks in reality, where tasks often have different power characteristics $[2,11,12]$. The power consumption function of a task running at the processor speed $s$ is of the form $h s^{\alpha}$, where $h$ and $\alpha$ are task and hardware dependent power characteristics, respectively $(0<h$ and $2 \leq \alpha \leq 3)$. The parameter setting of $h$ in $h s^{\alpha}$ might depend on the software implementation and the execution path of each task, whereas the value of $\alpha$ might depend on the hardware design of the processors under considerations. The objective of this paper is to minimize the energy consumption of task executions under the given deadline constraint. We are interested in the scheduling of frame-based task sets, in which all the tasks are ready at time 0 and share a common deadline. When task migration is allowed, we propose an optimal real-time task scheduling algorithm with a time complexity $O(|\mathbf{T}| \log |\mathbf{T}|)$ for multiprocessor environments, where $\mathbf{T}$ is the set of realtime tasks under considerations. When task migration is not allowed, a polynomial-time approximation algorithm is proposed. The approximation ratio of the algorithm is shown being 1.412. The performance of the approximation algorithm was evaluated by an extensive set of experiments, where excellent results were reported. 
The rest of this paper is organized as follows: In Section 2, we define the system models and formulate the problem. Section 3 presents an algorithm for multiprocessor energyefficient scheduling, when task migration is allowed. Our approximation algorithm to cope with systems which do not allow task migration is then presented in Section 4. Simulation results are shown in Section 5. Section 6 concludes this paper.

\section{Formal Models and Problem Definitions}

We are interested in energy-efficient scheduling of realtime tasks that are ready at time 0 and share a common deadline $D$ over multiple homogeneous processors. Each task $\tau_{i}$ is characterized by its worst-case execution CPU cycles $c_{i}$ and power consumption function $P_{i}()$ :

$$
P_{i}(s)=C_{i} V_{d d}^{2} s,
$$

where $s=\beta \frac{\left(V_{d d}-V_{t}\right)^{2}}{V_{d d}}$, and $s, C_{i}, V_{t}, V_{d d}$, and $\beta$ denote the processor speed, the effective switch capacitance, the threshold voltage (the minimum voltage that can be supplied to the processor for correct functionality), the supply voltage, and a hardware-design-specific constant, respectively $\left(V_{d d} \geq\right.$ $V_{t} \geq 0, \beta>0$, and $\left.C_{i}>0\right)$ [4, 17]. The value of the effective switch capacitance is highly related to the software implementations and the execution path of each task (which could be usually derived by profiling). Each power consumption function $P_{i}(s)$ can be phrased as $h_{i} \cdot s^{\alpha}$, where $\alpha$ is a hardware-dependent factor, and $h_{i}$ is a parameter related to the corresponding task execution $[3,9,12,15,19]$. For example, when $V_{t}$ is $0, h_{i}$ is $C_{i} / \beta^{2}$, and $\alpha$ is $3 . h_{i}$ is a positive real number, and $\alpha$ is usually a real number between between 2 and $3[12,15]$. It is clear that the power consumption function is a strictly convex and increasing function of the processor speed when the processor speeds are non-negative numbers. In this paper, we assume that $P_{i}(s)$ is second-order differentiable.

Suppose that each processor could operate at a speed in $[0, \infty]$, and the speed of each processor could be adjusted independently from each another. We assume that the number of CPU cycles executed in a time interval is linearly proportional to the processor speed, and that the energy consumed for a processor in the execution of a task at the processor speed $s$ for $t$ time units is the multiplication of its corresponding power consumption at the speed $s$ and $t$. Let the amount of CPU cycles completed for a task running at a speed $s$ for $t$ time units be the multiplication of $s$ and $t$. Assume that the time and energy overheads required on speed/voltage switching be negligible. Since $P_{i}(s) / s$ is also a strictly convex and increasing function, an optimal schedule must execute each task $\tau_{i} \in \mathbf{T}$ entirely at a selected speed $s_{i}[2,6]$. Specifically, executing task $\tau_{i}$ at the speed $s$ consumes $P_{i}(s) \frac{c_{i}}{s}$ amount of energy. The energy consumption function $E_{i}()$ of $\tau_{i}$ is defined as a function of the execution time $t_{i}$ of $\tau_{i}$ : $E_{i}\left(t_{i}\right)=P_{i}\left(\frac{c_{i}}{t_{i}}\right) t_{i}=h_{i} \cdot c_{i}^{\alpha} / t_{i}^{\alpha-1}$. Note that the energy consumption function of the execution time of $\tau_{i}$ is a strictly convex and decreasing function.

Problem Definition We consider energy-efficient scheduling with and without task migration in this work, where migration cost is assumed being negligible. No task is allowed to execute simultaneously on more than one processor. A schedule of a task set $\mathbf{T}$ is a mapping of the executions of the tasks in $\mathbf{T}$ to processors in the system with an assignment of processor speeds for the corresponding execution intervals of the tasks. A schedule is feasible if no task misses its deadline $D$, and no task is executed simultaneously on more than one processor. The energy consumption of a schedule $S$ is denoted as $\Phi(S)$ which is the sum of the energy consumption of task executions in $S$. A schedule is optimal if it is feasible, and its energy consumption is equal to the minimum energy consumption of all feasible schedules. Two energy-efficient scheduling problems are defined, as follows:

\section{Definition 1 Multiprocessor Energy-Efficient Scheduling with Task Migration (MEESM)}

Consider a set $\mathbf{T}$ of independent tasks over $M$ identical processors, where all tasks in $\mathbf{T}$ are ready at time 0 and share a common deadline $D$. Each task $\tau_{i} \in \mathbf{T}$ is associated with a computation requirement equal to $c_{i} \mathrm{CPU}$-cycles and a power consumption function $P_{i}()$ of a given processor speed. The objective is to derive a schedule for $\mathbf{T}$ such that all of the tasks in $\mathbf{T}$ complete before $D$, the total energy consumption is minimized, where task migration among processors is allowed.

A variation of the MEESM problem without task migration could be defined similarly as follows:

Definition 2 Multiprocessor Energy-Efficient Scheduling without Task Migration (MEES)

The input, output, and objective of this problem are as the same as their counterparts of the MEESM problem, where no task migration among processors is allowed.

If the number of tasks in $\mathbf{T}$ is no more than the number of processors in the system, the executing of each task $\tau_{i}$ on a different processor from time 0 to $D$ at the speed $\frac{c_{i}}{D}$ is an optimal schedule. For the rest of this paper, we only focus our discussions on the other case in which the number of tasks in $\mathbf{T}$ is more than the number of processors in the system. Since the MEES problem is $\mathcal{N} \mathcal{P}$-hard even when all of the power consumption functions of the tasks are the same [5], we propose an efficient algorithm which finds an approximated solution with a worst-case guarantee on the energy consumption by adopting approximation algorithms [16].

\section{An Optimal Algorithm When Task Migra- tion is Allowed}

In this section, we present an optimal algorithm for the MEESM problem. Since $P_{i}(s) / s$ is a convex and increasing 
function of the processor speed for every task $\tau_{i}$ in a given task set $\mathbf{T}$, there exists an optimal schedule that executes each task $\tau_{i} \in \mathbf{T}$ entirely at some speed $s_{i}[2,6]$. In the following discussions, we only consider schedules in which the entire duration of a task executes at the same speed.

Let $V=\left(t_{1}, t_{2}, \ldots, t_{|\mathbf{T}|}\right)$ be an assignment of execution times of tasks in $\mathbf{T}$, where $t_{i}$ is a positive real number for every task $\tau_{i} \in \mathbf{T}$. The energy consumption of an assignment $V$ of task execution times of $\mathbf{T}$ is defined as $\sum_{\tau_{i} \in \mathbf{T}} E_{i}\left(t_{i}\right) . V$ is said feasible for $\mathbf{T}$ on $M$ processors if the sum of execution times of all of the tasks in $\mathbf{T}$ is no greater than $M \cdot D$ (i.e., $\left.\sum_{\tau_{i} \in \mathbf{T}} t_{i} \leq M \cdot D\right)$, and $t_{i}$ is no greater than $D$ for every task $\tau_{i}$ in $\mathbf{T}$. Given a feasible schedule $S_{V}$ of $\mathbf{T}$, it is clear that a feasible assignment $V$ of task execution times can be derived by setting the execution time of task $\tau_{i}$ in $V$ as that of $\tau_{i}$ in $S_{V}$. The energy consumption of $V$ is equal to that of $S_{V}$. In the following lemma, we show that we can efficiently derive a feasible schedule $S_{V}$ with the same energy consumption as that of a given feasible assignment $V$ of execution times of tasks in $\mathbf{T}$. In other words, a feasible assignment $V$ of execution times of tasks in $\mathbf{T}$ with the minimum energy consumption leads to an optimal schedule of $\mathbf{T}$ for the MEESM problem.

Lemma 1 Given a feasible assignment $V$ of execution times of tasks in $\mathbf{T}$, a feasible schedule $S_{V}$ can be derived in $O(|\mathbf{T}|)$ such that the energy consumption of $S_{V}$ is equal to that of $V$.

Proof. We prove this lemma by constructing a feasible schedule $S_{V}$ according to $V=\left(t_{1}, t_{2}, \ldots, t_{|\mathbf{T}|}\right)$ :

- Case 1: If $\left\lceil\frac{\sum_{j=1}^{i-1} t_{j}}{D}\right\rceil=\left\lceil\frac{\sum_{j=1}^{i} t_{j}}{D}\right\rceil$, then execute $\tau_{i}$ on the $\left\lceil\frac{\sum_{j=1}^{i-1} t_{j}}{D}\right\rceil$-th processor from time $\left(\sum_{j=1}^{i-1} t_{j} \bmod D\right)$ to $\left(\sum_{j=1}^{i} t_{j}\right.$ $\bmod D)$.

- Case 2: If $\left\lceil\frac{\sum_{j=1}^{i-1} t_{j}}{D}\right\rceil \neq\left\lceil\frac{\sum_{j=1}^{i} t_{j}}{D}\right\rceil$, then execute $\tau_{i}$ on the $\left\lceil\frac{\sum_{j=1}^{i-1} t_{j}}{D}\right\rceil$-th processor from time $\left(\sum_{j=1}^{i-1} t_{j} \bmod D\right)$ to $D$ and on the $\left\lceil\frac{\sum_{j=1}^{i} t_{j}}{D}\right\rceil$-th processor from time 0 to $\left(\sum_{j=1}^{i} t_{j} \bmod D\right)$.

Since $0<t_{i} \leq D$ for all $\tau_{i}$ in $\mathbf{T}$ and $\sum_{\tau_{i} \in \mathbf{T}} t_{i}=M D$, the resulting schedule $S_{V}$ above is a feasible schedule for the MEESM problem. Besides, it is clear that the energy consumption of $S_{V}$ is equal to the energy consumption of $V$, since the execution time of task $\tau_{i}$ in $\mathbf{T}$ is $t_{i}$ both in $V$ and $S_{V}$. The time complexity is $O(|\mathbf{T}|)$ by taking the summation in an incremental manner.

Moreover, the strict convexity of the energy consumption functions of tasks would not allow any processor being idle between time 0 and time $D$ in an optimal schedule. In other words, an optimal schedule will always have some task executing between time 0 and time $D$ on any of the $M$ processors.
Lemma 2 When $|\mathbf{T}|>M$, there exists an optimal schedule which executes some task at any time instant between time 0 and time $D$ on each of the $M$ processors.

Proof. We prove this lemma by contradiction. Let $S_{V}$ be an optimal schedule, in which $S_{V}$ does not execute some task at some time instant between 0 and $D$ on at least one of the $M$ processors. Let $V=\left(t_{1}, t_{2}, \ldots, t_{|\mathbf{T}|}\right)$ be the assignment of execution times of $\mathbf{T}$ for schedule $S_{V}$. Therefore, we know that $\sum_{\tau_{i} \in \mathbf{T}} t_{i}<M \cdot D$. Since $|\mathbf{T}|>M$, there must be a task $\tau_{j}$ whose associated $t_{j}$ is less than $D$ in $V$. Stretching the execution time of $\tau_{j}$ as $\min \left\{D, M D-\sum_{\tau_{i} \in \mathbf{T} \backslash\left\{\tau_{j}\right\}} t_{i}\right\}$ results in a feasible assignment $V^{\prime}$ of execution times of tasks in $\mathbf{T}$. Because $E_{j}()$ is a strictly convex and decreasing function of the execution time of $\tau_{j}$, the energy consumption of $V^{\prime}$ is less than that of $V$. By Lemma 1, there exists a feasible schedule whose energy consumption is less than $S_{V}$, which contradicts the optimality of $S_{V}$. $\mathrm{T}$

Taking both Lemmas 1 and 2 into considerations at the same time, the MEESM problem can be formulated as a convex programming problem, as follows:

$$
\begin{array}{ll}
\text { minimize } & \sum_{\tau_{i} \in \mathbf{T}} E_{i}\left(t_{i}\right) \\
\text { subject to } & \sum_{\tau_{i} \in \mathbf{T}} t_{i}=M \cdot D \text { and } \\
& 0<t_{i} \leq D \quad \forall \tau_{i} \in \mathbf{T} .
\end{array}
$$

For the rest of this section, we will show that the optimal assignment of execution times of tasks in $\mathbf{T}$ described by Equation (2) can be determined in $O(|\mathbf{T}| \log |\mathbf{T}|)$ time by applying the Karush-Kuhn-Tucker optimality condition $[14, \S 14]$ and a binary search strategy. After the optimal assignment of execution times of tasks in $\mathbf{T}$ is determined, Lemma 1 is applied to derive an optimal schedule for the MEESM problem.

In the following, we first obtain an optimal solution by ignoring the condition $t_{i}>0$. After that, we show that $t_{i}>0$ is satisfied for every task $\tau_{i}$ in $\mathbf{T}$ for the solution. To apply the Karush-Kuhn-Tucker optimality condition for concave programming, Equation (2) is reformulated as a concave programming problem, as follows:

$$
\begin{array}{ll}
\text { maximize } & \sum_{\tau_{i} \in \mathbf{T}} \bar{E}_{i}\left(t_{i}\right) \\
\text { subject to } & \sum_{\tau_{i} \in \mathbf{T}} t_{i}=M \cdot D \text { and } \\
& t_{i} \leq D \quad \forall \tau_{i} \in \mathbf{T},
\end{array}
$$

where $\bar{E}_{i}\left(t_{i}\right)$ is defined as $-E_{i}\left(t_{i}\right)$. The Karush-KuhnTucker optimality condition for Equation (3) is to find a vector $\left(\lambda_{1}, \lambda_{2}, \ldots, \lambda_{|\mathbf{T}|}\right)$, a vector $\left(t_{1}^{*}, t_{2}^{*}, \ldots, t_{|\mathbf{T}|}^{*}\right)$, and a constant $\lambda$ such that

$$
\begin{gathered}
\bar{E}_{i}^{\prime}\left(t_{i}^{*}\right)-\lambda_{i}=\lambda, \quad t_{i}^{*} \leq D, \quad \forall \tau_{i} \in \mathbf{T}, \text { and } \\
\left(t_{i}^{*}-D\right) \lambda_{i}=0, \quad \lambda_{i} \geq 0, \\
\sum_{\tau_{i} \in \mathbf{T}} t_{i}^{*}=M D
\end{gathered}
$$

where $\bar{E}_{i}^{\prime}()$ is the derivative of $\bar{E}_{i}()$. Since $\sum_{\tau_{i} \in \mathbf{T}} \bar{E}_{i}\left(t_{i}\right)$ is a concave function, and $\left\{t_{j}\right\}$ is a quasiconvex set for every $\tau_{j} \in \mathbf{T}$, setting $\left(t_{1}, t_{2}, \ldots, t_{\mid \mathbf{T}} \mid\right)$ as $\left(t_{1}^{*}, t_{2}^{*}, \ldots, t_{|\mathbf{T}|}^{*}\right)$ is an 
optimal solution for the concave programming in Equation (3) $[14, \S 14]$. We will show that such a vector $\left(t_{1}^{*}, t_{2}^{*}, \ldots, t_{|\mathbf{T}|}^{*}\right)$ could be determined by the Lagrange multiplier technique. Let $\ell$ be an index, where $0 \leq \ell<M$. If the execution time of $\tau_{i}$ is set as $D$ for $i=1,2, \ldots, \ell$, the concave programming in Equation (3) could be rephrased as

$$
\begin{array}{ll}
\text { maximize } & \sum_{i=\ell+1}^{|\mathbf{T}|} \bar{E}_{i}\left(t_{i}\right) \\
\text { subject to } & \sum_{i=\ell+1}^{|\mathbf{T}|} t_{i}=(M-\ell) \cdot D
\end{array}
$$

by further ignoring the inequalities $t_{i} \leq D$ for $i=\ell+$ $1, \ldots,|\mathbf{T}|$.

Equation (5) could be solved by applying the Lagrange multiplier technique. Since $\bar{E}_{i}^{\prime}\left(t_{i}\right)=(\alpha-1) h_{i} c_{i}^{\alpha} t_{i}^{-\alpha}$, given an index $\ell$, the conditions $\bar{E}_{i}^{\prime}\left(t_{i}\right)=\bar{E}_{j}^{\prime}\left(t_{j}\right)$ for all $\ell<i, j \leq$ $|\mathbf{T}|$ for the Lagrange multiplier technique lead to

$$
\begin{gathered}
\frac{t_{i}^{\alpha}}{t_{j}^{\alpha}}=\frac{(\alpha-1) h_{i} c_{i}^{\alpha}}{(\alpha-1) h_{j} c_{j}^{\alpha}}, \quad \forall \ell<i, j \leq|\mathbf{T}| \\
\sum_{j=\ell+1}^{|\mathbf{T}|} t_{j}=(M-\ell) \cdot D .
\end{gathered}
$$

Therefore, the optimal solution for Equation (5) is to assign $\left(t_{\ell+1}, t_{\ell+2}, \ldots, t_{|\mathbf{T}|}\right)$ as $\left(t_{\ell+1}^{*}, t_{\ell+2}^{*}, \ldots, t_{|\mathbf{T}|}^{*}\right)$, where

$$
\begin{gathered}
\sum_{j=\ell+1}^{|\mathbf{T}|} t_{\ell+1}^{*} \frac{c_{j}}{c_{\ell+1}}\left(\frac{h_{j}}{h_{\ell+1}}\right)^{1 / \alpha}=(M-\ell) D \\
t_{j}^{*}=t_{\ell+1}^{*} \frac{c_{j}}{c_{\ell+1}}\left(\frac{h_{j}}{h_{\ell+1}}\right)^{1 / \alpha}, \forall \ell+1<j \leq|\mathbf{T}|,
\end{gathered}
$$

and the Lagrange multiplier $\lambda^{*}$ is $\bar{E}_{\ell+1}^{\prime}\left(t_{\ell+1}^{*}\right)$. As a result, the time complexity to derive the optimal solution of Equation (5) for an index $\ell$ is $O(|\mathbf{T}|-\ell)$.

It is clear that every $t_{j}^{*}$ in $\left(t_{\ell+1}^{*}, t_{\ell+2}^{*}, \ldots, t_{|\mathbf{T}|}^{*}\right)$ derived from Equation (6) is greater than 0. Therefore, if each $t_{j}^{*}$ in $\left(t_{\ell+1}^{*}, t_{\ell+2}^{*}, \ldots, t_{|\mathbf{T}|}^{*}\right)$ is no greater than $D$ when $\ell=0$, then assigning $t_{i}$ as $t_{i}^{*}$ for $\tau_{i}$ in $\mathbf{T}$ is an assignment of execution times with the minimum energy consumption. Therefore, we only have to consider the other case. For the rest of this section, let $\mathbf{T}$ be sorted by a non-decreasing order of $E_{i}^{\prime}(D)$, where $E_{i}^{\prime}()$ is the derivative of the energy consumption function $E_{i}()$. The following lemma helps to construct an assignment of execution times of tasks in $\mathbf{T}$ with the minimum energy consumption.

Lemma 3 Suppose that every $t_{j}^{*}$ in $\left(t_{\ell^{*}+1}^{*}, t_{\ell^{*}+2}^{*}, \ldots, t_{|\mathbf{T}|}^{*}\right)$ derived from Equation (6) is less than $D$ for an index $\ell^{*}$, and $\bar{E}_{\ell^{*}}^{\prime}(D)$ is no less than $\bar{E}_{\ell^{*}+1}^{\prime}\left(t_{\ell^{*}+1}^{*}\right)$, where $1 \leq \ell^{*}<M$. The assignment of $t_{i}$ as $D$, for $i=1,2, \ldots, \ell^{*}$, and $t_{j}$ as $t_{j}^{*}$, for $j=\ell^{*}+1, \ell^{*}+2, \ldots,|\mathbf{T}|$, would derive an assignment of task execution times with the minimum energy consumption.

Proof. We prove this lemma by showing that all of the conditions in Equation (4) hold. It is clear that such an assignment satisfies $\sum_{\tau_{i} \in \mathbf{T}} t_{i}^{*}=M D$ and $0<t_{i}^{*} \leq D$ for all $\tau_{i}$ in $\mathbf{T}$. Let $\lambda$ be $\bar{E}_{\ell^{*}+1}^{\prime}\left(t_{\ell^{*}+1}^{*}\right)$. For $j=\ell^{*}+1, \ell^{*}+2, \ldots,|\mathbf{T}|$, let $\lambda_{j}$ be 0 . For $i=1,2, \ldots, \ell^{*}$, let $\lambda_{i}$ be $\bar{E}_{i}^{\prime}(D)-\bar{E}_{\ell^{*}+1}^{\prime}\left(t_{\ell^{*}+1}^{*}\right)$.

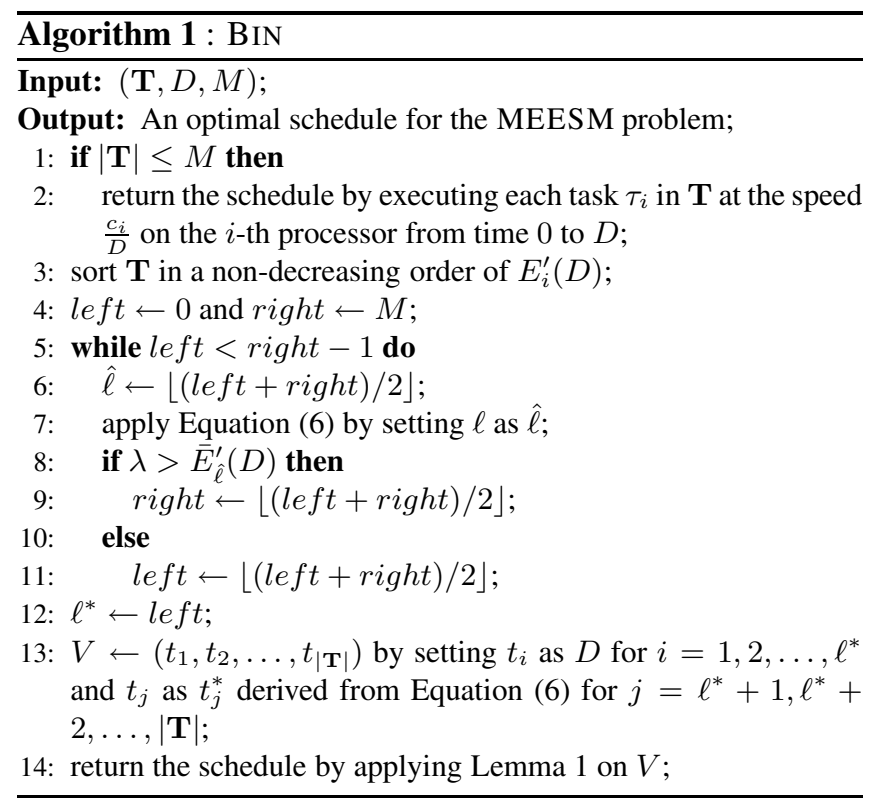

As a result, the equality $\bar{E}_{i}^{\prime}\left(t_{i}^{*}\right)-\lambda_{i}=\lambda$ holds for every task $\tau_{i}$ in $\mathbf{T}$. Since $\mathbf{T}$ is sorted in a non-decreasing order of $E_{i}^{\prime}(D)$, and $\bar{E}_{i}()$ is defined as $-E_{i}()$, we know that $\bar{E}_{i}^{\prime}(D) \geq \bar{E}_{j}^{\prime}(D)$ when $i<j$. Because of the condition $\bar{E}_{\ell^{*}}^{\prime}(D) \geq \bar{E}_{\ell^{*}+1}^{\prime}\left(t_{\ell^{*}+1}^{*}\right)$, we have

$$
\bar{E}_{i}^{\prime}(D) \geq \bar{E}_{\ell^{*}}^{\prime}(D) \geq \bar{E}_{\ell^{*}+1}^{\prime}\left(t_{\ell^{*}+1}^{*}\right)
$$

for any $i=1,2, \ldots, \ell^{*}$. As a result, $\lambda_{i}=\bar{E}_{i}^{\prime}(D)-$ $\bar{E}_{\ell^{*}+1}^{\prime}\left(t_{\ell^{*}+1}^{*}\right) \geq 0$ for $i=1,2, \ldots, \ell^{*}$. It is clear that all of the conditions in Equation (4) hold.

By Lemma 3, an assignment of execution times with the minimum energy consumption for the MEESM problem can be derived in $O(M|\mathbf{T}|+|\mathbf{T}| \log |\mathbf{T}|)$ by setting $\ell$ from 0 to $M-1$ sequentially. Moreover, the following lemma helps the reducing of the time complexity to $O(|\mathbf{T}| \log |\mathbf{T}|)$ by a binary search on the setting of $\ell$.

Lemma 4 Suppose that every $t_{j}^{*}$ in $\left(t_{\ell^{*}+1}^{*}, t_{\ell^{*}+2}^{*}, \ldots, t_{|\mathbf{T}|}^{*}\right)$ derived from Equation (6) is less than $D$ for an index $\ell^{*}$, and $\bar{E}_{\ell^{*}}^{\prime}(D)$ is no less than $\bar{E}_{\ell^{*}+1}^{\prime}\left(t_{\ell^{*}+1}^{*}\right)$, where $1 \leq \ell^{*}<M$. If $\ell^{*}<\hat{\ell}<M$, the Lagrange multiplier for Equation (5) by setting $\ell$ as $\hat{\ell}$ is strictly greater than $\bar{E}_{\hat{\ell}}^{\prime}(D)$. If $\hat{\ell} \leq \ell^{*}$, the Lagrange multiplier for Equation (5) by setting $\ell$ as $\hat{\ell}$ is no greater than $\bar{E}_{\hat{\ell}}^{\prime}(D)$.

Proof. For notational brevity, let $\bar{t}_{\hat{\ell}+1}$ and $\bar{t}_{\hat{\ell}}$ be the values of $t_{\hat{\ell}+1}^{*}$ and $t_{\hat{\ell}}^{*}$ derived from Equation (6) by setting $\ell$ as $\ell^{*}$, respectively. We consider the case when $\ell^{*}<\hat{\ell}<M$. As shown in Lemma 3 , we know $\bar{E}_{\hat{\ell}+1}^{\prime}\left(\bar{t}_{\hat{\ell}+1}\right)=\bar{E}_{\hat{\ell}}^{\prime}\left(\bar{t}_{\hat{\ell}}\right)$. When $\ell$ is set as $\hat{\ell}$, one can verify that $t_{\hat{\ell}+1}^{*}$ derived from Equation (6) is strictly less than $\bar{t}_{\hat{\ell}+1}$. Since $\bar{E}_{j}^{\prime}()$ is a decreasing function of the execution time for any task $\tau_{j}$ in $\mathbf{T}$, we know

$$
\left.\lambda^{*}=\bar{E}_{\hat{\ell}+1}^{\prime}\left(t_{\hat{\ell}+1}^{*}\right)>\bar{E}_{\hat{\ell}+1}^{\prime}\left(\bar{t}_{\hat{\ell}+1}\right)=\bar{E}_{\hat{\ell}}^{\prime} \bar{t}_{\hat{\ell}}\right)>\bar{E}_{\hat{\ell}}^{\prime}(D),
$$


where $\lambda^{*}$ is the Lagrange multiplier for Equation (5) by setting $\ell$ as $\hat{\ell}$, and the last inequality comes from the condition $\bar{t}_{\hat{\ell}}<D$. The other case can also be proved in a similar manner. $\square$

Our proposed algorithm denoted as Algorithm BIN (shown in Algorithm 1) adopts the binary search strategy. After all, we conclude this section by showing the following theorem.

Theorem 1 Algorithm BIN can derive an optimal schedule for the MEESM problem in $O(|\mathbf{T}| \log |\mathbf{T}|)$.

Proof. It follows directly from Lemmas 1, 2, and 4.

\section{An Approximation Algorithm When Task Migration is not Allowed}

In this section, we present an approximation algorithm for the MEES problem. Since the flexibility of task migration relaxes the constraint on the dis-allowance of task migration for the MEES problem, the energy consumption of the optimal schedule for the MEESM problem is no more than that of the optimal schedule for the MEES problem for the same task set $\mathbf{T}$ on $M$ processors. Our proposed approximation algorithm first estimates a lower bound on the minimum energy consumption for the MEES problem by applying Algorithm BIN (presented in Section 3). Then, a feasible schedule of the MEES problem is derived by referring to the optimal schedule of the MEESM problem.

For the rest of this paper, let $t_{i}^{*}$ denote the estimated execution time of task $\tau_{i}$ in $\mathbf{T}$, which is defined as the execution time of $\tau_{i}$ in the optimal solution derived from Algorithm BIN when task migration is allowed. The estimated execution times of tasks in $\mathbf{T}$ are then used to assign tasks onto these $M$ processors. Let $e_{i}^{*}$ be the estimated energy consumption of task $\tau_{i}$ when the execution time of $\tau_{i}$ is the estimated execution time of $\tau_{i}$, i.e., $e_{i}^{*}=E_{i}\left(t_{i}^{*}\right)$. Let $p_{m}$ denote the load on the $m$-th processor. The load of a processor is defined as the total amount of estimated execution time of the tasks assigned onto this processor. For notational brevity, let $T_{m}$ denote the set of the tasks assigned onto the $m$-th processor. Our proposed algorithm shown in Algorithm 2 (denoted as Algorithm LEET) adopts the Largest-Estimated-ExecutionTime-First strategy. That is, tasks are considered in a nonincreasing order of their estimated execution time.

For notational brevity, for the rest of this paper, let $\mathbf{T}$ be a sorted set in a non-increasing order of the estimated execution time, i.e., $t_{i}^{*} \geq t_{j}^{*}$ if $i<j$. Algorithm LEET considers the tasks in the sorted order from $\tau_{1}$ to $\tau_{|\mathbf{T}|}$. Once task $\tau_{i}$ is considered, $\tau_{i}$ is assigned onto the $m$-th processor whose current load is the smallest. (For the simplicity on presentation, we break ties by choosing the smallest index $m$. Actually, the analysis in the following still holds by breaking ties arbitrarily.) After the assignment of the tasks onto the $M$ processors is done, we have to assign the execution times of these tasks to meet the timing constraint. For every task $\tau_{i}$ assigned onto the $m$-th processor, the execution time of $\tau_{i}$ is set as $t_{i}^{*} \frac{D}{p_{m}}$. After all, it is clear that the total execution time of the tasks assigned onto each processor is exactly equal to $D$. Since the processor speed is in $[0, \infty]$, executing the tasks assigned onto each processor one after one is a feasible schedule of the MEES problem. The time complexity of Algorithm LEET is $O(|\mathbf{T}| \log |\mathbf{T}|)$, which is dominated by applying Algorithm BIN, the sorting of the tasks, and the procedure to find the minimum $p_{m}$.

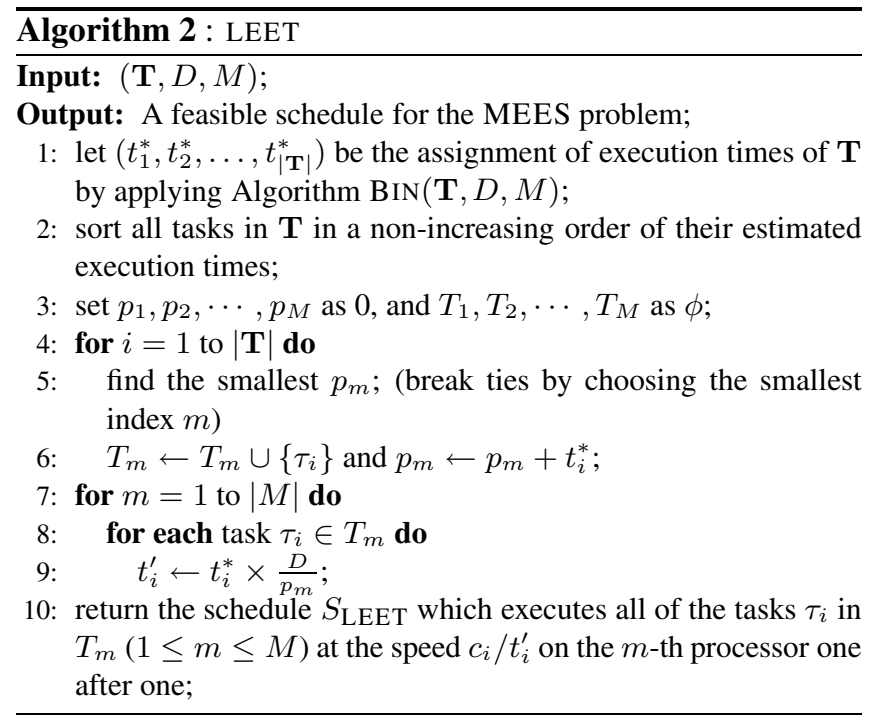

In the following, we shall show the optimality of Algorithm LEET, i.e., that on the approximation ratio. For the simplicity of representation, the schedule derived from Algorithm LEET is denoted as $S_{\text {LEET }}$ for task set $\mathbf{T}$. For notational brevity, let $\mathbf{T}^{\prime}$ be a subset of $\mathbf{T}$, where $\mathbf{T}^{\prime}$ consists of those tasks whose estimated execution times are strictly less than $D$. That is, $\mathbf{T}^{\prime}=\left\{\tau_{i} \mid t_{i}^{*}<D, \forall \tau_{i} \in \mathbf{T}\right\}$. Moreover, let $\hat{\mathbf{T}}$ be the difference set of $\mathbf{T}$ from $\mathbf{T}^{\prime}$, i.e., $\hat{\mathbf{T}}=\mathbf{T} \backslash \mathbf{T}^{\prime}$. Note that the analysis in the rest of this section only focuses on the case that $|\mathbf{T}|>M$ since Algorithm LEET guarantees to derive an optimal schedule for the other case. Furthermore, this also implies that $\mathbf{T}^{\prime}$ is not empty for the rest discussions. We will show that the approximation ratio of Algorithm LEET is $\frac{(\alpha-1)^{\alpha-1}\left(2^{\alpha}-1\right)^{\alpha}}{\alpha^{\alpha}\left(2^{\alpha}-2\right)^{\alpha-1}}$. Since the value of $\alpha$ is at most 3 , and the approximation ratio is an increasing function on the value of $\alpha$, the approximation ratio of Algorithm LEET is 1.412 .

Before we proceed to prove the approximation ratio of Algorithm LEET, we first introduce some properties of the estimated energy consumptions and the estimated execution times of the tasks under considerations. In the following lemma, we show that the ratio of the estimated energy consumption to the estimated execution time of task $\tau_{i}$ is equal to that of task $\tau_{j}$ if both $\tau_{i}$ and $\tau_{j}$ are elements of $\mathbf{T}^{\prime}$.

Lemma 5 For any two tasks $\tau_{i}, \tau_{j} \in \mathbf{T}^{\prime}$, the ratio of the estimated energy consumption to the estimated execution time of 
$\tau_{i}$ is equal to that of $\tau_{j}$, i.e., $\frac{e_{i}^{*}}{t_{i}^{*}}=\frac{e_{j}^{*}}{t_{j}^{*}}$.

Proof. It follows directly from the property of the Lagrange multiplier. By the definition of the task set $\mathbf{T}^{\prime}, t_{i}^{*}$ and $t_{j}^{*}$ are both less than $D$. By Lemma 3 and Algorithm BIN, we have $-E_{i}^{\prime}\left(t_{i}^{*}\right)=-E_{j}^{\prime}\left(t_{j}^{*}\right)$. Therefore, we conclude this lemma by showing that

$$
\begin{gathered}
\quad-(\alpha-1) h_{i} \frac{c_{i}^{\alpha}}{\left(t_{i}^{*}\right)^{\alpha}}=-(\alpha-1) h_{j} \frac{c_{j}^{\alpha}}{\left(t_{j}^{*}\right)^{\alpha}} \\
\Rightarrow \quad \frac{t_{i}^{*}}{t_{j}^{*}}=\frac{\left(t_{j}^{*}\right)^{\alpha-1} h_{i} c_{i}^{\alpha}}{\left(t_{i}^{*}\right)^{\alpha-1} h_{j} c_{j}^{\alpha}}=\frac{e_{i}^{*}}{e_{j}^{*}} .
\end{gathered}
$$

口

If two processors are only assigned with some tasks in $\mathbf{T}^{\prime}$ after all of the tasks are assigned in Algorithm LEET, we show that the ratio of the loads between these two processors is at most 2 .

Lemma 6 Suppose that the $m^{*}$-th and the $\hat{m}$-th processors are assigned with some tasks in $\mathbf{T}^{\prime}$ such that $p_{m^{*}}$ is the maximum and $p_{\hat{m}}$ is the minimum after all of the tasks are assigned onto processors in Algorithm LEET, then $p_{m^{*}}$ is at most twice of $p_{\hat{m}}$.

Proof. Since tasks are assigned onto the processor with the smallest load, and $|\mathbf{T}|>M$, it is clear that both $p_{m^{*}}$ and $p_{\hat{m}}$ are greater than 0 . For each task $\tau_{i}$ in $\hat{\mathbf{T}}$, Algorithm LEET assigns only $\tau_{i}$ onto a processor. Therefore, $\sum_{\tau_{i} \in \mathbf{T}^{\prime}} t_{i}^{*}=(M-|\hat{\mathbf{T}}|) D$. Since $\left|\mathbf{T}^{\prime}\right|>(M-|\hat{\mathbf{T}}|)$, we know $p_{m^{*}} \geq D$ and $p_{\hat{m}} \leq D$ by the pigeon hole principle. Because of the condition $t_{i}^{*}<D$ for every task $\tau_{i}$ in $\mathbf{T}^{\prime}, T_{m^{*}}$ consists of at least two tasks. Let the last task inserted into $T_{m^{*}}$ be $\tau_{r}$. When $\tau_{r}$ is considered in the first loop in Algorithm LEET (i.e., the for loop from Steps 4 to 6), there must be at least one task assigned onto the $\hat{m}$-th processor already, since $\tau_{r}$ is assigned onto the processor whose current load is the minimum. Let $\tau_{q}$ be the first task assigned onto the $\hat{m}$ th processor. Because Algorithm LEET assigns the tasks in a non-increasing order of the estimated execution times, we have $t_{r}^{*} \leq t_{q}^{*} \leq p_{\hat{m}}$. Furthermore, since $\tau_{r}$ is assigned onto the processor whose current load is the minimum, we know $p_{m^{*}}-t_{r}^{*} \leq p_{\hat{m}}$. By considering the above inequalities, we know $p_{m^{*}} \leq 2 p_{\hat{m}}$. $\square$

Besides, we need the following lemma to prove the approximation ratio of Algorithm LEET.

Lemma 7 Suppose $f(x)=k \cdot(2 x)^{\alpha}+(\hat{M}-k) x^{\alpha}$ for a positive number $\hat{M}$ and a non-negative number $k$, where $0 \leq$ $k \leq \hat{M}$ and $2 k \cdot x+(\hat{M}-k) \cdot x=\hat{M}$, then

$$
f(x) \leq \frac{(\alpha-1)^{\alpha-1}\left(2^{\alpha}-1\right)^{\alpha}}{\alpha^{\alpha}\left(2^{\alpha}-2\right)^{\alpha-1}} \hat{M}
$$

Proof. Since $2 k \cdot x+(\hat{M}-k) \cdot x=\hat{M}$, we know $k=\frac{\hat{M}-\hat{M} x}{x}$. Therefore,

$$
f(x)=\hat{M}\left(x^{\alpha-1}\left(2^{\alpha}-1\right)+x^{\alpha}\left(2-2^{\alpha}\right)\right),
$$

and the derivative of $f(x)$ is

$$
f^{\prime}(x)=\hat{M}\left((\alpha-1) x^{\alpha-2}\left(2^{\alpha}-1\right)+\alpha x^{\alpha-1}\left(2-2^{\alpha}\right)\right) .
$$

$f(x)$ is maximized at $x^{*}$ when $f^{\prime}\left(x^{*}\right)=0$. By solving $f^{\prime}\left(x^{*}\right)=0$, we have $x^{*}=\frac{(\alpha-1)\left(2^{\alpha}-1\right)}{\alpha\left(2^{\alpha}-2\right)}$. As a result, we conclude that

$$
f(x) \leq f\left(x^{*}\right)=\frac{(\alpha-1)^{\alpha-1}\left(2^{\alpha}-1\right)^{\alpha}}{\alpha^{\alpha}\left(2^{\alpha}-2\right)^{\alpha-1}} \hat{M} .
$$

$\square$

We conclude this section by showing that Algorithm LEET is a $\frac{(\alpha-1)^{\alpha-1}\left(2^{\alpha}-1\right)^{\alpha}}{\alpha^{\alpha}\left(2^{\alpha}-2\right)^{\alpha-1}}$-approximation algorithm for the MEES problem.

Theorem 2 The approximation ratio of Algorithm LEET is $\frac{(\alpha-1)^{\alpha-1}\left(2^{\alpha}-1\right)^{\alpha}}{\alpha^{\alpha}\left(2^{\alpha}-2\right)^{\alpha-1}}$.

Proof. Let $O^{*}$ be the energy consumption for an optimal schedule for $\mathbf{T}$ of the MEES problem. Since the sum of the estimated energy consumption of all of the tasks in $\mathbf{T}$ is a lower bound on $O^{*}$, we know that $O^{*} \geq \sum_{\tau_{i} \in \mathbf{T}} e_{i}^{*}$. Let $\tau_{r}$ be some task in $\mathbf{T}^{\prime}$. By Lemma 5, we have

$$
O^{*} \geq \sum_{\tau_{i} \in \mathbf{T}} e_{i}^{*}=e_{r}^{*} / t_{r}^{*}(M-|\hat{\mathbf{T}}|) D+\sum_{\tau_{i} \in \hat{\mathbf{T}}} e_{i}^{*} .
$$

For each task $\tau_{i}$ in $\hat{\mathbf{T}}$, Algorithm LEET assigns only $\tau_{i}$ onto a processor. Since we break ties by choosing the smallest index $m$ in Algorithm LEET, the $i$-th processor is assigned with only task $\tau_{i}$, where $0<i \leq|\hat{\mathbf{T}}|$. For the $m$-th processor, where $m>|\hat{\mathbf{T}}|$, the energy consumption to execute task $\tau_{i}$ in $T_{m}$ is equal to $e_{i}^{*}\left(\frac{p_{m}}{D}\right)^{\alpha-1}$ in $S_{\text {LEET }}$, and the sum of the estimated energy consumption of the tasks in $T_{m}$ is equal to $\frac{e_{r}^{*}}{t_{r}^{*}} p_{m}$ by applying Lemma 5. Therefore, we have

$$
\begin{aligned}
\Phi\left(S_{\text {LEET }}\right) & =\sum_{\tau_{i} \in \hat{\mathbf{T}}} e_{i}^{*}+\sum_{m=|\hat{\mathbf{T}}|+1}^{M} \frac{e_{r}^{*}}{t_{r}^{*}} p_{m}\left(\frac{p_{m}}{D}\right)^{\alpha-1} \\
& =\sum_{\tau_{i} \in \hat{\mathbf{T}}} e_{i}^{*}+\sum_{m=|\hat{\mathbf{T}}|+1}^{M} \frac{e_{r}^{*}}{t_{r}^{*}}\left(\frac{p_{m}}{D}\right)^{\alpha} D .
\end{aligned}
$$

The approximation ratio $\mathcal{A}$ of Algorithm LEET can be phrased as

$$
\mathcal{A}=\frac{\Phi\left(S_{\text {LEET }}\right)}{O^{*}} \leq \frac{\sum_{m=|\hat{\mathbf{T}}|+1}^{M}\left(\frac{p_{m}}{D}\right)^{\alpha}}{M-|\hat{\mathbf{T}}|},
$$

where the inequality comes from the fact $\frac{a+b_{1}}{a+b_{2}} \leq \frac{b_{1}}{b_{2}}$ when $b_{1} \geq b_{2}>0$ and $a \geq 0$. It remains to show that

$$
\frac{\sum_{m=|\hat{\mathbf{T}}|+1}^{M}\left(\frac{p_{m}}{D}\right)^{\alpha}}{M-|\hat{\mathbf{T}}|} \leq \frac{(\alpha-1)^{\alpha-1}\left(2^{\alpha}-1\right)^{\alpha}}{\alpha^{\alpha}\left(2^{\alpha}-2\right)^{\alpha-1}}
$$

Suppose that the $m^{*}$-th and the $\hat{m}$-th processors are assigned with some tasks in $\mathbf{T}^{\prime}$ such that $p_{m^{*}}$ is the maximum, and 
$p_{\hat{m}}$ is the minimum. By Lemma 6, we have $2 \frac{p_{\hat{m}}}{D} \geq \frac{p_{m^{*}}}{D} \geq$ $\frac{p_{m}}{D} \geq \frac{p_{\hat{m}}}{D}$, for all $|\hat{\mathbf{T}}|<m \leq M$. Besides, by the convexity of $\left(\frac{p_{m}}{D}\right)^{\alpha}$ of $\frac{p_{m}}{D}$ (i.e., the second order derivative of $\left(\frac{p_{m}}{D}\right)^{\alpha}$ of $\frac{p_{m}}{D}$ is non-negative when $\frac{p_{m}}{D} \geq 0$ ) and the fact $p_{\hat{m}} \geq$ $2 p_{\hat{m}}-p_{m} \geq 0$ for all $|\hat{\mathbf{T}}|<m \leq M$, we have

$$
\left(\frac{p_{m}}{D}\right)^{\alpha} \leq \frac{2 p_{\hat{m}}-p_{m}}{p_{\hat{m}}}\left(\frac{p_{\hat{m}}}{D}\right)^{\alpha}+\left(1-\frac{2 p_{\hat{m}}-p_{m}}{p_{\hat{m}}}\right)\left(\frac{2 p_{\hat{m}}}{D}\right)^{\alpha},
$$

since $\frac{2 p_{\hat{m}}-p_{m}}{p_{\hat{m}}}\left(\frac{p_{\hat{m}}}{D}\right)+\left(1-\frac{2 p_{\hat{m}}-p_{m}}{p_{\hat{m}}}\right)\left(\frac{2 p_{\hat{m}}}{D}\right)$ is equal to $\frac{p_{m}}{D}$. Therefore,

$$
\begin{aligned}
\sum_{m=|\hat{\mathbf{T}}|+1}^{M}\left(\frac{p_{m}}{D}\right)^{\alpha} & \leq \sum_{m=|\hat{\mathbf{T}}|+1}^{M}+\frac{\frac{2 p_{\hat{m}}-p_{m}}{p_{\hat{m}_{m}}}\left(\frac{p_{\hat{m}}}{D}\right)^{\alpha}}{\left(1-\frac{2 p_{\hat{m}}-p_{m}}{p_{\hat{m}}}\right)\left(\frac{2 p_{\hat{m}}}{D}\right)^{\alpha}} \\
& =k \cdot\left(\frac{2 p_{\hat{m}}}{D}\right)^{\alpha}+(M-|\hat{\mathbf{T}}|-k)\left(\frac{p_{\hat{m}}}{D}\right)^{\alpha},
\end{aligned}
$$

where $2 k \frac{p_{\hat{m}}}{D}+(M-|\hat{\mathbf{T}}|-k) \frac{p_{\hat{m}}}{D}=(M-|\hat{\mathbf{T}}|)$, i.e., $k=\sum_{m=|\hat{\mathbf{T}}|+1}^{M}\left(1-\frac{2 p_{\hat{m}}-p_{m}}{p_{\hat{m}}}\right)$. By applying Lemma 7 with the setting of $\hat{M}$ as $M-|\hat{\mathbf{T}}|$ and $x$ as $\frac{p_{\hat{m}}}{D}$, we reach the conclusion.

Corollary 1 The approximation ratio of Algorithm LEET is 1.412 .

Proof. Since $\alpha$ is no greater than 3 , and $\frac{(\alpha-1)^{\alpha-1}\left(2^{\alpha}-1\right)^{\alpha}}{\alpha^{\alpha}\left(2^{\alpha}-2\right)^{\alpha-1}}$ is an increasing function of $\alpha$, the approximation ratio is bounded when $\alpha=3$.

\section{Performance Evaluation}

In this section, we provide performance evaluation on the energy consumption of Algorithm LEET. Another algorithm, denoted as Algorithm RAND, which is very similar to Algorithm LEET, was simulated for comparison. The only difference between Algorithm RAND and Algorithm LEET is that tasks are not sorted before the assignment procedure in Algorithm RAND.

Workload Parameters and Performance Metrics The common deadline $D$ of the tasks in a task set was set as 100 units of time in the simulations. For each task $\tau_{i}$ in $\mathbf{T}, \tau_{i}$ was characterized by two different parameters: the number of execution CPU cycles $c_{i}$ and the coefficient $h_{i}$ of the power consumption function of $\tau_{i} . c_{i}$ was generated uniformly in the range $(0, D] . h_{i}$ was uniformly distributed in the range of 2 and 10. The exponent of the power consumption functions of the processor speed $s$ was set as 3, i.e., $P_{i}(s)=h_{i} s^{3}$. We simulated two cases for different numbers of processors with different numbers of tasks. For the first case, we evaluated the algorithms for the effects on the ratio of the number of tasks to the number of processors. For a given ratio $\eta$ of the number of tasks to the number of processors, the number of processors $M$ was an integral random variable between 10 and 30 , and

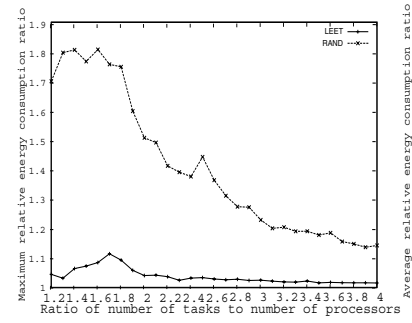

(a) Maximum ratio when $\alpha=3$

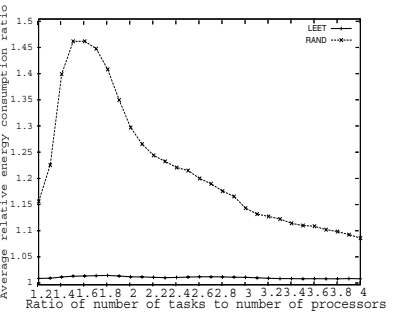

(b) Average ratio when $\alpha=3$

\section{Figure 1. (a) and (b): maximum and average rel- ative energy consumption ratios, respectively.}

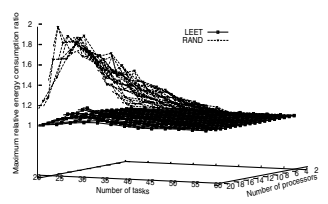

(a) Maximum ratio when $\alpha=3$

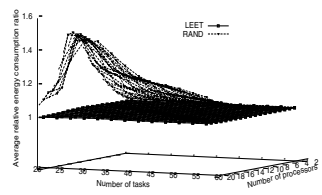

(b) Average ratio when $\alpha=3$
Figure 2. (a) and (b): maximum and average relative energy consumption ratios, respectively.

the number of tasks was set as the floor of the multiplication of $\eta$ and $M$, i.e., $\lfloor\eta \cdot M\rfloor$. For the other case, the number of processors ranged from 2 to 20 , and the task-set size ranged from 21 to 60 . Experimental results were conducted with 512 independent experiments for each parameter configuration.

The relative energy consumption ratio was adopted as the performance metric in our experiments. The relative energy consumption ratio for an input instance was defined as the energy consumption of the schedule derived by the algorithm to that of an optimal schedule with the allowance of task migration. As shown in Section 3, the energy consumption of an optimal schedule with the allowance of task migration can be derived in an efficient manner. Since the problem is $\mathcal{N} \mathcal{P}$ hard, the performance metric relative energy consumption ratio aimed at the providing of an approximated index. When the results were for the average relative energy consumption ratio, their results were averaged. When they were for the maximum relative energy consumption ratio, the maximum value was returned.

Experimental Results For the evaluation of the effects on the ratio of the number of tasks to the number of processors, Figures 1(a) and 1(b) present the maximum and average relative energy consumption ratios for the simulated algorithms. The performance of Algorithm LEET was very close to that 
of the optimal solutions. The maximum and average relative energy consumption ratios for Algorithm LEET were less than 1.11 and 1.01, respectively. Furthermore, the maximum and average relative energy consumption ratios for Algorithm RAND were less than 1.82 and 1.46 , respectively. When the ratio of the number of tasks to the number of processors was small, both of Algorithm LEET and Algorithm RAND might assign a task along with improper tasks on a processor. Such an assignment might result in a significant increase on the energy consumption of these tasks when the energy consumption for the other tasks were almost as the same as that in the optimal schedule. Such an observation explained why the maximum relative energy consumption ratios in Figure 1(a) decreased when the ratio of the number of tasks to the number of processors increased. However, when the ratio of the number of tasks to the number of processors was small, in most cases, most processors were assigned with only one task, and the assignment was almost as the same as that of an optimal schedule. Therefore, the average energy consumption ratio was relatively small when the ratio of the number of tasks to the number of processors was less than 1.5.

When the number of processors ranged from 2 to 20 , and the task set size ranged from 21 to 60 , Figure 2(a) (/Figure 2(b)) presents the maximum (/average) relative energy consumption ratios for the simulated algorithms. The performance of Algorithm LEET was again very close to the optimal solution. The maximum and average relative energy consumption ratios for Algorithm LEET were less than 1.084 and 1.01 respectively, where the maximum and average relative energy consumption ratios for Algorithm RAND were less than 1.941 and 1.485 , respectively. The trends of the simulation results were similar to those in Figures 1(a) and 1(b). The results indicated that Algorithm LEET could derive effective schedules for the MEES problem.

\section{Conclusion}

This paper targets energy-efficient scheduling problems over homogeneous processors for real-time tasks with a common deadline. Different from the past work, we consider different parameter settings for the power consumption function $P_{i}(s)$ of the processor speed $s$ for each task $\tau_{i}$, i.e., $P_{i}(s)=h_{i} s^{\alpha}$, where the value of $h_{i}$ depends upon the power characteristics of task $\tau_{i}$ and $\alpha$ is a hardware-specific constant $(2 \leq \alpha \leq 3)$. We propose an optimal algorithm, when task migration is permitted, and an approximation algorithm, when task migration is not allowed. We show that the approximation ratio of the proposed approximation algorithm is $\frac{(\alpha-1)^{\alpha-1}\left(2^{\alpha}-1\right)^{\alpha}}{\alpha^{\alpha}\left(2^{\alpha}-2\right)^{\alpha-1}}$. Since the value of $\alpha$ is at most 3 , the approximation ratio is at most 1.412 . The proposed algorithm is evaluated by a series of simulation experiments, compared to a lower bound by allowing task migration. The performance of Algorithm LEET is very close to that of the optimal solutions. The results also indicate that Algorithm LEET could derive effective schedules for the MEES problem.

For future research, we will explore energy-efficient scheduling over multiple processors for periodic real-time tasks or tasks with arbitrary deadlines and arrival times.

\section{References}

[1] J. H. Anderson and S. K. Baruah. Energy-efficient synthesis of periodic task systems upon identical multiprocessor platforms. In Proceedings of the 24th International Conference on Distributed Computing Systems, pages 428-435, 2004.

[2] H. Aydin, R. Melhem, D. Mossé, and P. Mejía-Alvarez. Determining optimal processor speeds for periodic real-time tasks with different power characteristics. In Proceedings of the IEEE EuroMicro Conference on Real-Time Systems, page 225, 2001.

[3] N. Bansal, T. Kimbrel, and K. Pruhs. Dynamic speed scaling to manage energy and temperature. In Proceedings of the 2004 Symposium on Foundations of Computer Science, pages 520-529, 2004.

[4] A. Chandrakasan, S. Sheng, and R. Broderson. Lower-power CMOS digital design. IEEE Journal of Solid-State Circuit, 27(4):473-484, 1992.

[5] J.-J. Chen, H.-R. Hsu, K.-H. Chuang, C.-L. Yang, and A.-C. P. T.W. Kuo. Multiprocessor energy-efficient scheduling with task migration considerations. In EuroMicro Conference on Real-Time Systems (ECRTS'04), pages 101-108, 2004.

[6] J.-J. Chen, T.-W. Kuo, and C.-L. Yang. Profit-driven uniprocessor scheduling with energy and timing constraints. In ACM Symposium on Applied Computing, pages 834-840. ACM Press, 2004.

[7] F. Gruian. System-level design methods for low-energy architectures containing variable voltage processors. In Power-Aware Computing Systems, pages 1-12, 2000.

[8] F. Gruian and K. Kuchcinski. Lenes: Task scheduling for low energy systems using variable supply voltage processors. In Proceedings of Asia South Pacific Design Automation Conference, pages 449-455, 2001.

[9] S. Irani, S. Shukla, and R. Gupta. Algorithms for power savings. In Proceedings of the Fourteenth Annual ACM-SIAM Symposium on Discrete Algorithms, pages 37-46. Society for Industrial and Applied Mathematics, 2003.

[10] T. Ishihara and H. Yasuura. Voltage scheduling problems for dynamically variable voltage processors. In Proceedings of the International Symposium on Low Power Electroncs and Design, pages 197-202, 1998.

[11] W.-C. Kwon and T. Kim. Optimal voltage allocation techniques for dynamically variable voltage processors. In Proceedings of the 40th Design Automation Conference, pages 125-130, 2003.

[12] P. Mejía-Alvarez, E. Levner, and D. Mossé. Adaptive scheduling server for power-aware real-time tasks. ACM Transactions on Embedded Computing Systems, 3(2):284-306, 2004.

[13] R. Mishra, N. Rastogi, D. Zhu, D. Mossé, and R. Melhem. Energy aware scheduling for distributed real-time systems. In International Parallel and Distributed Processing Symposium, page 21, 2003.

[14] R. L. Rardin. Optimization in Operations Research. Prentice Hall, 1998.

[15] Y. Shin and K. Choi. Power conscious fixed priority scheduling for hard real-time systems. In Proceedings of the 36th ACM/IEEE Conference on Design Automation Conference, pages 134-139, 1999.

[16] V. V. Vazirani. Approximation Algorithms. Springer, 2001.

[17] M. Weiser, B. Welch, A. Demers, and S. Shenker. Scheduling for reduced CPU energy. In Proceedings of Symposium on Operating Systems Design and Implementation, pages 13-23, 1994.

[18] C.-Y. Yang, J.-J. Chen, and T.-W. Kuo. An approximation algorithm for energy-efficient scheduling on a chip multiprocessor. In Proceedings of the 8th Conference of Design, Automation, and Test in Europe (DATE), pages 468-473, 2005.

[19] F. Yao, A. Demers, and S. Shenker. A scheduling model for reduced CPU energy. In Proceedings of the 36th Annual Symposium on Foundations of Computer Science, pages 374-382. IEEE, 1995.

[20] Y. Zhang, X. Hu, and D. Z. Chen. Task scheduling and voltage selection for energy minimization. In Annual ACM IEEE Design Automation Conference, pages 183-188, 2002. 